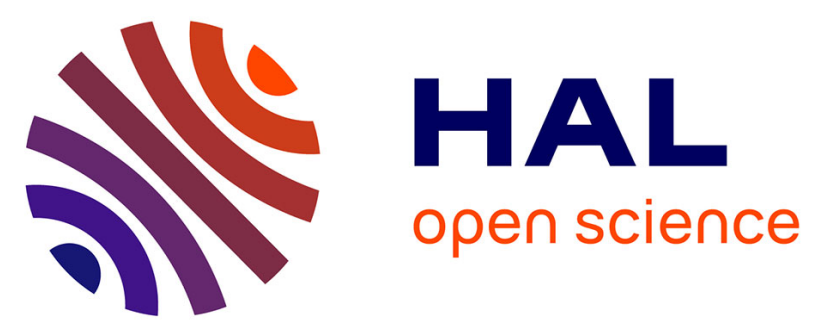

\title{
La série lithique du " Pendu" à Étaples-sur-Mer (estuaire de la Canche, Pas-de-Calais) : une occupation à la transition Néolithique ancien/moyen
}

\author{
Marie Bouchet, Laurent Deschodt, Frédéric Lemaire
}

\section{- To cite this version:}

Marie Bouchet, Laurent Deschodt, Frédéric Lemaire. La série lithique du "Pendu " à Étaples-surMer (estuaire de la Canche, Pas-de-Calais) : une occupation à la transition Néolithique ancien/moyen. Bulletin de la Société préhistorique française, 2012, 109 (3), pp.571-575. 10.3406/bspf.2012.14177. hal-02055126

\section{HAL Id: hal-02055126 https://hal-inrap.archives-ouvertes.fr/hal-02055126}

Submitted on 3 Mar 2019

HAL is a multi-disciplinary open access archive for the deposit and dissemination of scientific research documents, whether they are published or not. The documents may come from teaching and research institutions in France or abroad, or from public or private research centers.
L'archive ouverte pluridisciplinaire HAL, est destinée au dépôt et à la diffusion de documents scientifiques de niveau recherche, publiés ou non, émanant des établissements d'enseignement et de recherche français ou étrangers, des laboratoires publics ou privés. 


\title{
ACTUALITÉS SCIENTIFIQUES
}

\author{
DÉCOUVERTES RÉCENTES
}

\section{La série lithique du «Pendu » à Étaples-sur-Mer (estuaire de la Canche, Pas-de-Calais) : une oc- cupation à la transition Néolithique ancien/ moyen}

\section{Contexte}

La région d'Étaples, avec notamment le site des «Sablins », est un secteur clef de la Préhistoire régionale (Piningre et al., 1991; Philippe et al., 2005 et 2011). Des diagnostics réalisés à proximité (Bostyn et Martial, 1992; Bostyn, 2006; Deschodt et al., 2011) confirment la présence sur la bordure nord de l'estuaire d'un paléosol holocène avec du matériel lithique sous une couverture sableuse.

En 2009, un diagnostic en bordure de plateau a permis de mettre en évidence, au lieu-dit «Le Pendu» (fig. 1), un nouveau gisement (Lemaire et al., 2009). Le plateau crayeux domine l'estuaire d'environ $25 \mathrm{~m}$. Le contact s'effectue par un versant en pente forte, voire par de petits escarpements. Le gisement, exploré en puits, occupe un petit vallon élémentaire qui rejoint directement l'estuaire.

\section{Stratigraphie}

Le talweg incisé dans la craie est partiellement comblé par un lœss (unité 4, fig. 1) Le dépôt est attribuable à la fin du Pléniglaciaire weichselien (Antoine, 2002; Koster, 2005). Il est pédogenisé en partie supérieure (6). Un horizon humifère (7) se développe sur le lœss. Il contient le matériel archéologique et constituait l'ancienne surface topographique avant le dépôt de sable (8). La date 5950 $\pm 30 \mathrm{BP}$ obtenue sur un charbon dans le sol offre un terminus post quem pour l'enfouissement de l'horizon et est concordante avec l'attribution chronologique du matériel fouillé (voir ci-dessous).

Le versant du talweg exposé à l'ouest est nappé de sable, reprise éolienne de sédiments marins. Le dépôt s'effectue postérieurement au sol (7) en deux épisodes distincts (8 et 10) séparés par une pédogenèse (9). La stratigraphie est corrélable avec celle des «Sablins», où l'épaisseur est moindre et le paléosol moins marqué (Piningre et al., 1991; Philippe et al., 2005). Les datations radiocarbone qui encadrent le premier dépôt de sable permettent de proposer une fourchette chronologique large, concordant avec l'attribution chronoculturelle du matériel. La comparaison avec les séquences paléoenvironnementales régionales définies sur le littoral par M. Meurisse-Fort (2008) permet de corréler le dépôt brusque et abondant du sable avec la transgression atlantique qui culmine au début du IV millénaire. Le dépôt de sable est antérieur à environ 3900 av. J.-C., période de régression marine.

Le second dépôt éolien (10) est postérieur au début de la romanisation de la région (présence d'une fibule de la période augustéenne dans le sol 9).

\section{Vestiges}

Le sondage SP9 a livré, à plus de $2 \mathrm{~m}$ de profondeur, des artefacts en abondance. Une fouille manuelle a été effectuée sur une fenêtre d'environ $8 \mathrm{~m}^{2}$. Une petite concentration de silex taillés se développe sur environ $2 \mathrm{~m}^{2}$. Elle est associée à une zone charbonneuse d'environ $50 \mathrm{~cm}$ de diamètre et épaisse de $5 \mathrm{~cm}$.

La série lithique se compose de 310 artefacts $(7,5 \mathrm{~kg})$ en bon état général de conservation. La matière première utilisée est exclusivement le silex crétacé local gris-noir (Sénonien) présent sous la forme de galets marins et de rognons issus d'affleurements. La production est nettement orientée vers le débitage d'éclats. Quatre-vingt-dix éclats courts ont été relevés ainsi que douze éclats laminaires (fig. 2, nos 6 à 8). Il faut ajouter deux outils réalisés sur ce type de support : un burin sur éclat retouché (fig. 3, $\mathrm{n}^{\circ}$ 9) et un denticulé (fig. 2, $\mathrm{n}^{\circ} 2$ ). L'outillage comporte aussi des pièces réalisées sur de gros éclats tels qu'une pièce esquillée et un denticulé. On notera la présence de neuf lamelles (fig. 2, nos 3 et 4). Des pièces bifaciales sont également présentes au sein du corpus : il s'agit d'un 
$0 \quad$ échelle verticale $=3 \times$ éch. horizontale

1
2
3
4
5
6
7
8
9

$4220 \pm 30 \mathrm{BP}$

charbon de bois (charcoal)

Nord-Ouest

Lyon-7710

SP2

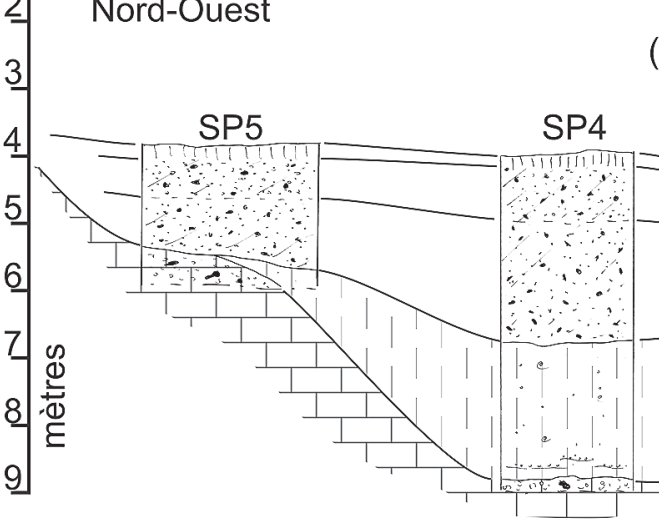

SacA 22654)

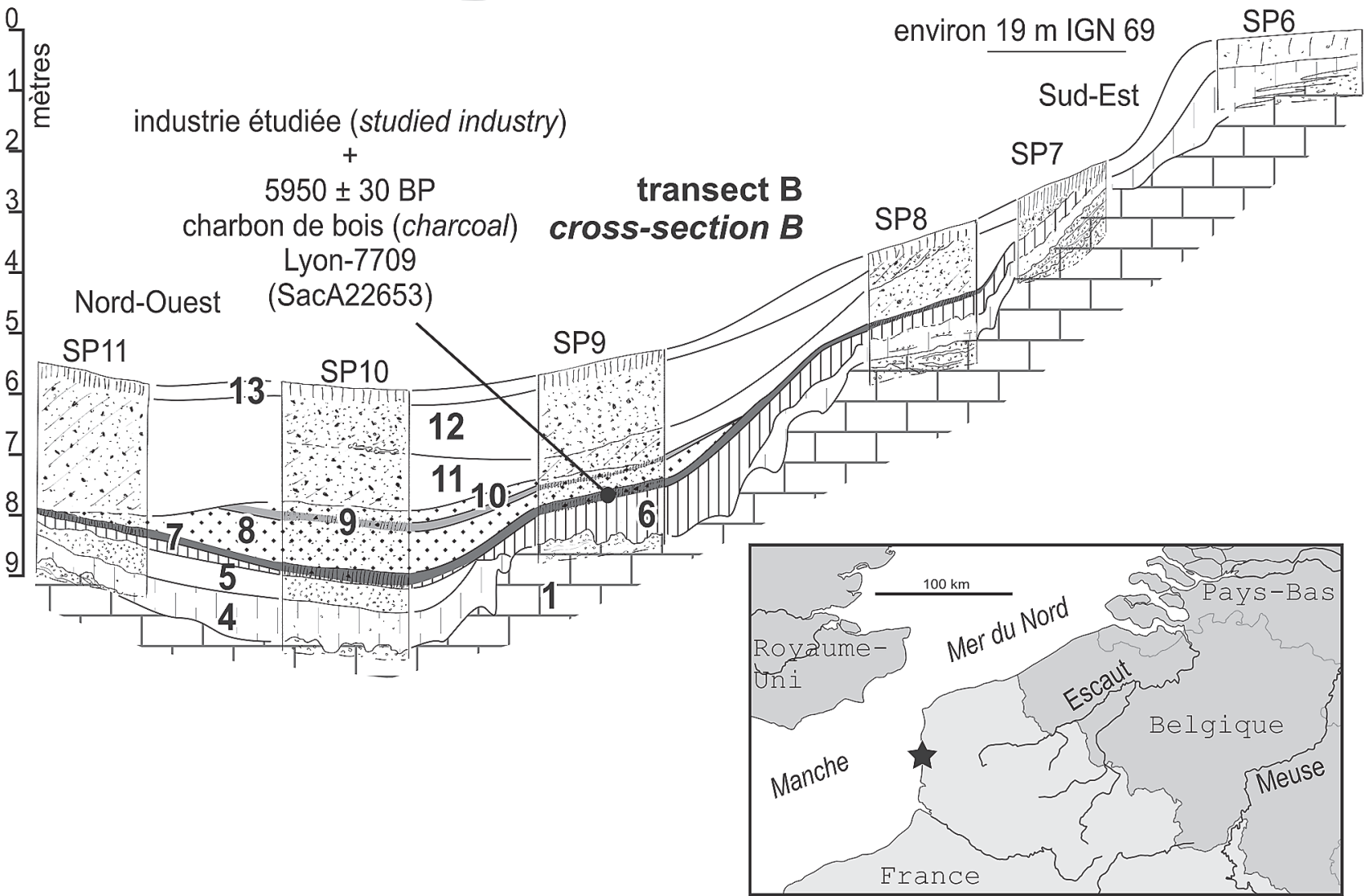

$\exists \quad$ échelle verticale $=$ éch. horizontale (transect $B$ )

vertical scale $=$ horizontal scale $\quad($ cross-section $B)$

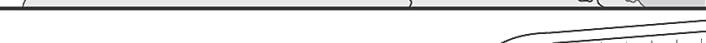

transect A

11

4

cross-section A

$6]_{1}^{7}$

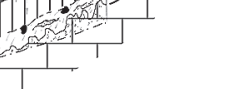
27

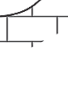



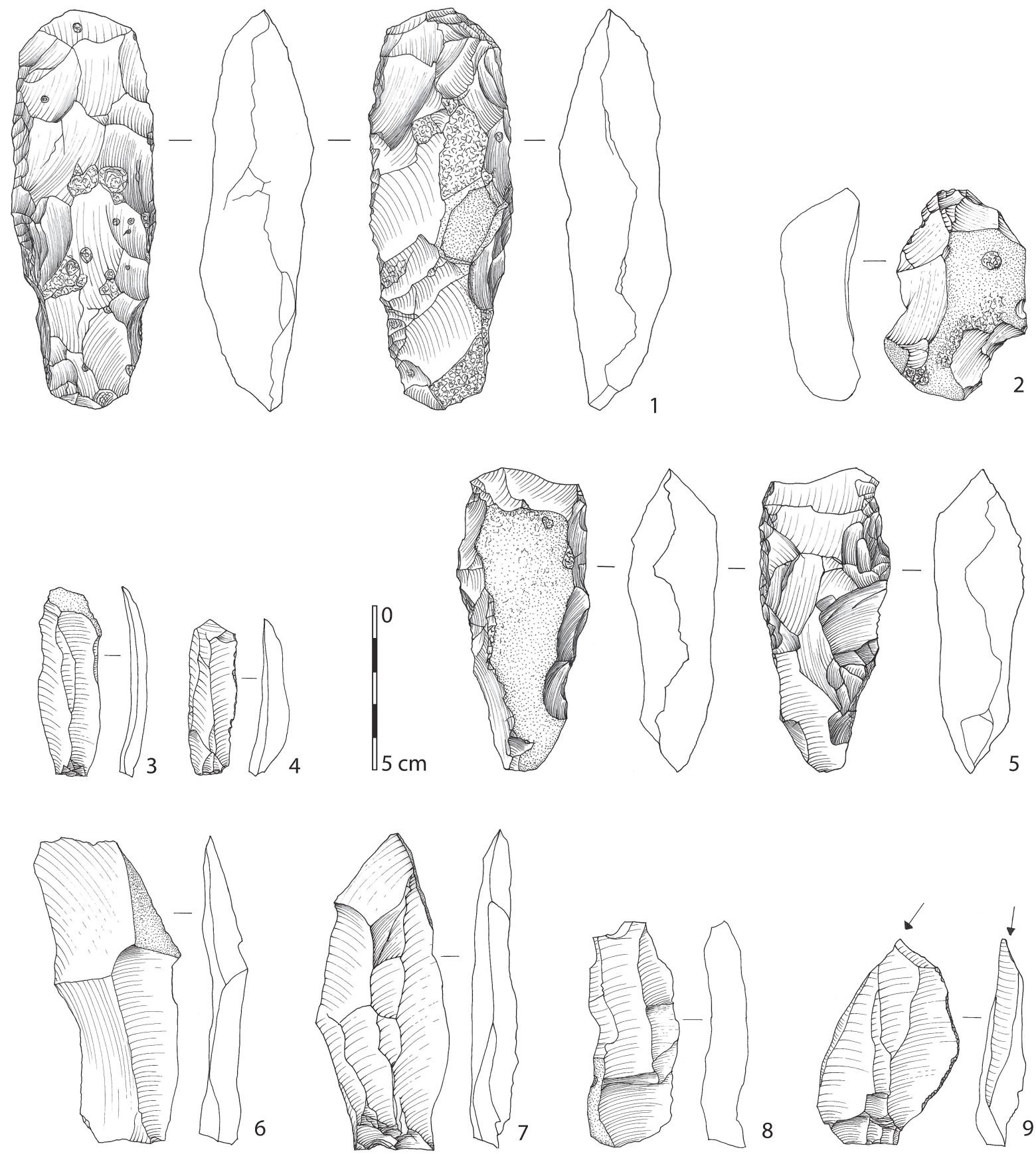

Fig. 2 - Industrie lithique. $1:$ hache; $2:$ denticulé sur éclat; 3 et $4:$ lamelles ; $5:$ tranchet; 6,7 et $8:$ éclats laminaires; $9:$ burin sur éclat retouché (dessins J. Lantoine).

tranchet (fig. 2, $\mathrm{n}^{\circ} 5$ ) et d'une hache de plus de $10 \mathrm{~cm} \mathrm{de}$ long (fig. $2, \mathrm{n}^{\circ} 1$ ). Des éléments reflètent des activités de débitage et de façonnage : neuf nucléus dont un nucléus retouché et un nucléus réutilisé en percuteur, un rognon testé, un galet testé, un percuteur, 11 éclats d'entame, 81 déchets et 89 esquilles.

Les lamelles ont été réalisées au percuteur tendre par percussion indirecte alors que les éclats laminaires ont été obtenus au percuteur dur, par percussion directe. Les nucleus reflètent deux organisations de débitage différentes : unipolaire et multidirectionnel.

Ces vestiges témoignent de la présence d'une zone d'activités comprenant au moins une aire de débitage et un foyer. La représentation de toute la chaîne opératoire étaye cette idée. D'autre part, la présence d'une molette, de quelques petits fragments de céramique et du foyer permettent d'envisager une occupation proche.

\section{Comparaison avec le site des «Sablins», attribution chronologique et commentaires}

Cette découverte est à comparer avec le site des «Sablins» qui a fait l'objet de plusieurs campagnes de fouille programmée (Hurtrelle et Piningre, 1976; Piningre et al., 1991; Philippe et al., 2005, 2006, 2007, 2009 et 2011). Ces dernières ont mis en évidence une succession d'occupations datées du Néolithique moyen I et II et, de 
manière plus ténue, du Néolithique final. Concernant la période la plus ancienne, de nombreux ateliers de taille du silex ont été mis au jour ainsi que des structures de combustion, des fosses, un puits, des trous de poteau et de piquet. Des superpositions témoignent d'occupations successives mais l'attribution chronoculturelle reste peu claire. Si les industries lithique et céramique présentent des caractères indéniablement rattachables au Néolithique moyen I Cerny, certains éléments donnent un aspect plus ancien à rapprocher de la culture Villeneuve-SaintGermain/Bliquy. Il est possible que le site livre une phase VSG/Blicquy suivie d'une phase Cerny mais la fouille n'a pas permis de différencier ces occupations. Il se peut également que le site présente une phase ancienne du Cerny, héritant de traditions des cultures précédentes, comme le suggèrent des tessons avec association de décors d'affinité postrubanée (VSG/Blicquy) et Cerny (Piningre et al., 1991; Philippe et al., 2009). La récente découverte, dans un niveau daté du Néolithique moyen I, d'un vase entier cassé sur place dont la typologie est rattachable au Néolithique moyen II (Philippe et al., 2011) conforte l'hypothèse d'un palimpseste.

Pour l'heure, seules deux datations ${ }^{14} \mathrm{C}$ sont disponibles pour ces occupations anciennes. Elles ont été obtenues à partir de charbons de bois provenant de deux fosses (Hurtrelle et Piningre, 1978) : Gif 3701, $5660 \pm 120$ BP, et Gif 4024, $5690 \pm 120 \mathrm{BP}$, soit respectivement 6476 $\pm 130 \mathrm{cal} \mathrm{BP}$ et $6501 \pm 130 \mathrm{cal} \mathrm{BP}(1 \delta$, CalPal 2007 HULU); soit une fourchette commune entre 4681 et 4396 av. J.-C. (1 $\delta)$.

Les vestiges du «Pendu» ne reflètent qu'un échantillon du mobilier du site identifié. Cependant, même à ce stade des recherches, de fortes similitudes avec les occupations anciennes du site des «Sablins» sont apparentes. Outre la configuration stratigraphique sensiblement proche, l'industrie lithique est analogue, tant par la matière première que par le débitage et l'outillage. La céramique comporte de l'os pour dégraissant comme la céramique des groupes VSG/Blicquy et Cerny. Enfin, la concentration de silex taillés s'apparente à certaines structures découvertes aux «Sablins».

Le petit corpus du «Pendu» peut difficilement apporter des précisions chronoculturelles. La datation du foyer (Ly 7709, $5950 \pm 30 \mathrm{BP}$, soit $6790 \pm 62 \mathrm{cal} \mathrm{BP}-1 \delta$, CalPal 2007 HULU - soit 4882 à 4798 av. J.-C., fig. 1) est légèrement plus haute que celles du site des «Sablins», cependant elle est compatible avec l'industrie mise au jour. Nous proposons un âge à la transition entre le Néolithique ancien et moyen. La possibilité de la présence d'une industrie appartenant au Néolithique ancien aux «Sablins»a été soulevée sans pouvoir être confirmée; d'autre part les niveaux de sol comportant les amas de taille n'ont pas encore été datés. S'il convient d'être prudent avec cette seule date radiocarbone, le gisement $\mathrm{du}$ «Pendu» pourrait toutefois témoigner de l'ancienneté des implantations néolithiques dans la partie occidentale du Nord-Pas-de-Calais.

\section{Conclusion}

Dans le Nord-Pas-de-Calais, à la différence du Bassin parisien et du Hainaut belge occidental, aucun témoin n'a pu être observé concernant les initiateurs du courant de néolithisation. Seule la présence de la culture VSGBlicquy est attestée, mais les vestiges sont très rares et se concentrent sur un petit territoire entre la Deûle et la Scarpe. Une fouille préventive réalisée en 2006 à Loison-sous-Lens a permis l'étude d'un habitat (deux unités identifiables et une sépulture, Praud et al., 2010). Enfin, deux occupations ont été partiellement observées sur les communes de Noyelles-sous-Lens et de Vitry-enArtois lors de la fouille d'une villa pour la première et d'un diagnostic pour la seconde (Hosdez, 2001; Maniez, 2006). Dans l'état actuel des données, l'estuaire de la Canche témoignerait donc des plus anciennes sociétés agro-pastorales dans la partie occidentale du Nord-Pasde-Calais.

Remerciements à Françoise Bostyn pour ses conseils.

\section{RÉFÉRENCES BIBLIOGRAPHIQUES}

ANTOINE P. (2002) - Les lœss en France et dans le Nord-Ouest européen, Revue française de géotechnique, 99, p. 3-21

BOSTYN F. (2006) - Étaples-sur-Mer «RN 39» (Pas-de-Calais), rapport de diagnostic, INRAP, Villeneuve-d'Ascq, service régional de l'Archéologie du Nord - Pas-de-Calais, 16 p.

BOSTYN F., MARTIAL E. (1992) - Étaples-sur-mer «RN $39 »$ (Pasde-Calais) Aldi, rapport de diagnostic, INARP, Villeneuve-d'Ascq, service régional de l'Archéologie du Nord - Pas-de-Calais, 16 p.

DESCHODT L., LORIN Y., LECOMTE-SCMITT B., MARTIAL E. (2011) - Étaples (Pas-de-Calais), ZAC Opalopolis, RD 939 : occupations anciennes sur les coteaux de l'estuaire de la Canche, rapport de diagnostic, INRAP, Amiens, service régional de l'Archéologie du Nord - Pas-de-Calais, 95 p.

HOSDEZ C. (2001) - Noyelles-sous-Lens (62), parcelle Bertelsman Services, rapport de fouille, INRAP, Villeneuve-d'Ascq, service régional de l'Archéologie du Nord - Pas-de-Calais, 40 p.

HURTRELLE J., PININGRE J.-F. (1976) - Le site néolithique des Sablins à Étaples (Pas-de-Calais), premiers résultats, Septentrion, 6, p. $46-57$.

HURTRELLE J., PININGRE J.-F. (1978) - Datation radiocarbone du Cerny des Sablins à Étaples (Pas-de-Calais), Bulletin de la Société préhistorique française, 75, 3, p. 83-86.

KOSTER E. A. (2005) - Recent Advances in Luminescence Dating of Late Pleistocene (Cold-Climate) Aeolian Sand and Loess Deposits in Western Europe, Permafrost and Periglacial Processes, 16, 1, p. 131-143.

LEMAIRE F., BOUCHET M., DESCHODT L., MARIETTE E., TORON S. (2009) - Étaples (Pas-de-Calais), ZAC du domaine du Chemin des Près, rapport de diagnostic, INRAP, Amiens, service régional de l'Archéologie du Nord - Pas-de-Calais, 129 p.

MANIEZ J. (2006) - Vitry-en-Artois, chemin brûlé (62), rapport de diagnostic, DACAD-INRAP, Villeneuve-d'Ascq, service régional de l'Archéologie du Nord - Pas-de-Calais, 36 p.

MEURISSE-FORT M. (2008) - Enregistrement haute résolution des massifs dunaires. Manche, mer du Nord et Atlantique. Le rôle des tempêtes, Paris, Publibook Université, 306 p.

PHILLIPPE M., MEURISSE M., RASSART V., RASSAT S. (2005) Sondage archéologiques et morphosédimentaires sur le site d'Étaples "Les Sablins »(Pas-de-Calais), Août-septembre 2004, document final de synthèse, Villeneuve-d'Ascq, service régional de l'Archéologie du Nord - Pas-de-Calais, 27 p.

PHILIPPE M., MARCH R., MEURISSE M., RASSART V. (2006) Étaples «Les Sablins» (Pas-de-Calais), fouilles juillet 2005, document final de synthèse, Villeneuve-d'Ascq, service régional de l'Archéologie du Nord - Pas-de-Calais, 42 p. 
PHILIPPE M. CASPAR J.P., GOSSELIN G., MARCH R. MEURISSE M., PITON D., RASSART V. (2007) - Étaples «Les Sablins» (Pas-de-Calais), campagne 2006, rapport de fouilles, Villeneuve-d'Ascq, service régional de l'Archéologie du Nord - Pasde-Calais, 67 p. et annexes.

PHILIPPE M, RASSART V., GOSSELIN G., MEURISSE M. RASSAT S. (2009) - Étaples «Les Sablins» (Pas-de-Calais), rapport de fouilles programmées, campagne 2008, Villeneuve-d'Ascq, service régional de l'Archéologie du Nord - Pas-de-Calais, 35 p.

PHILIPPE M., RASSART V., COHEN C., GOSSELIN G., GUERET C., LEROY-LAFAURIE P., MEURISSE-FORT M., OUESLATI T., RASSAT S., WIRTZ B. (2011) - Etaples «Les Sablins» (Pas-deCalais), rapport de fouilles programmées, campagne 2009 et postfouille 2009/2011, Villeneuve-d'Ascq, service régional de l'Archéologie du Nord - Pas-de-Calais, 73 p.

PININGRE J.-F., BOSTYN F., COUPPE J. (1991) - L'atelier de taille du silex des Sablins à Étaples (Pas-de-Calais), Gallia Préhistoire, 33, p. 83-135.

PRAUD I., BOSTYN F., CAYOL N., HAMON C., LADUREAU P., LANCHON Y., PINARD E., (2010) - Entre Blicquy et VilleneuveSaint-Germain : présentation de la fouille d'un habitat du Néolithique ancien à Loison-sous-Lens (Pas-de-Calais), in C. Billard et M. Legris (dir.), Premiers Néolithiques de l'Ouest : cultures, réseaux, échanges des premières sociétés néolithiques à leur expansion, actes du Colloque interrégional sur le Néolithique (Le Havre, 2007), Rennes, PUR (Archéologie et culture), p. 305-323.

\section{Marie BOUCHET}

INRAP Méditerranée

561, rue Étienne-Lenoir, Km Delta, 30900 Nîmes marie.bouchet@inrap.fr

Laurent DESCHODT

INRAP Nord-Picardie, ZI de la Pilaterie 11, rue des Champs, 59650 Villeneuve-d'Ascq LGP-UMR 8591

laurent.deschodt@inrap.fr

Frédéric LEMAIRE

INRAP Nord-Picardie

7, rue Pascal, 62217 Achicourt frederic.lemaire@inrap.fr 